\title{
QPOs in cataclysmic variables and in X-ray binaries
}

\author{
W. Kluźniak ${ }^{1,2,3}$, J.-P. Lasota ${ }^{1,4}$, M. A. Abramowicz ${ }^{1,5, \star}$, and B. Warner ${ }^{6}$ \\ 1 Nordita, Blegdamsvej 17, 2100 Copenhagen, Denmark \\ e-mail: lasota@iap.fr \\ 2 Institute of Astronomy, Univ. of Zielona Góra, ul. Lubuska 2, 65-265 Zielona Góra, Poland \\ ${ }^{3}$ Copernicus Astronomical Center, ul. Bartycka 18, 00-716 Warszawa, Poland \\ 4 Institut d'Astrophysique de Paris, UMR 7095 CNRS, Univ. P. \& M. Curie, 98bis Bd. Arago, 75014 Paris, France \\ 5 Department of Astrophysics, Göteborg University, Göteborg, Sweden \\ ${ }^{6}$ Department of Astronomy, University of Cape Town, Rondebosch 7700, South Africa
}

Received 4 May 2005 / Accepted 29 July 2005

\begin{abstract}
Recent observations, reported by Warner and Woudt, of Dwarf Nova Oscillations (DNOs) exhibiting frequency drift, period doubling, and 1:2:3 harmonic structure, can be understood as disc oscillations that are excited by perturbations at the spin frequency of the white dwarf or of its equatorial layers. Similar quasi-periodic disc oscillations in black hole low-mass X-ray binary (LMXB) transients in a 2:3 frequency ratio show no evidence of frequency drift and correspond to two separate modes of disc oscillation excited by an internal resonance. Just as no effects of general relativity play a role in white dwarf DNOs, no stellar surface or magnetic field effects need be invoked to explain the black hole QPOs.
\end{abstract}

Key words. accretion, accretion discs - novae, cataclysmic variables - X-ray: binaries

\section{QPO similarities and differences between white dwarfs and black holes}

The highest frequencies of nearly periodic modulations of X-rays (HF QPOs) in black holes and neutron stars (van der Klis 2000, and references therein) attract a great deal of attention because it is thought that their frequencies reflect dynamic phenomena in the motion of matter in strong-field gravity. Disc oscillations in Einstein's gravity are a favored explanation (Kato 2001; Wagoner 1999), and a 2:3 ratio of frequencies has been pointed out by Abramowicz \& Kluźniak (2001) and suggested to be a manifestation of non-linear internal resonance of accretion discs.

Recently, Warner \& Woudt (2005a, and references therein) have discovered that in dwarf nova outbursts in VW Hyi, the DNO oscillations (sub-Hz dwarf nova oscillations reminiscent of HF QPOs in neutron stars and black holes) exhibit period doubling and tripling. At times two or three frequencies in a $1: 2: 3$ ratio are present at the same time in the light curve. At first sight this seems analogous to the frequencies reported in black holes (e.g., the frequencies in a 1:2:3 ratio in the source XTE J1550-564; Remillard et al. 2002), and led to attempts at finding a common model for DNOs in accreting white dwarfs and QPOs in candidate black holes (Warner \& Woudt 2005a). However, while the two phenomena are similar, they are not identical.

^ Theoretical Physics, Chalmers University, 412-96 Göteborg, Sweden.
The differences are two-fold. In black holes binaries the twin HF QPOs have fixed frequencies, within errors of several percent (McClintock \& Remillard 2004), while the DNO frequencies vary with time (as the luminosity decreases) by a factor exceeding two. Further, in white dwarf DNOs, on occasion, two or more variable frequencies are present simultaneously, always in a 1:2:3 ratio, i.e., more than one harmonic is present. In black holes, usually only one mode of oscillation is manifest at a given time. Although sometimes both HF QPO frequencies are present at the same time in some black holes (Strohmayer 2001; Remillard et al. 2002), careful analysis reveals that the two frequencies are not harmonics of one non-sinusoidal oscillation (McClintock \& Remillard 2004).

We suggest that the DNOs can be interpreted in terms of resonant disk oscillations invoked to explain the HF QPOs in black hole binaries (Abramowicz \& Kluźniak 2001; Kluźniak \& Abramowicz 2003), but with another excitation mechanism that is related to the difference between black holes and white dwarfs. White dwarfs have a surface which can, e.g., support a magnetic field, and is capable of disturbing the disc strictly periodically at a well-defined rotation rate. None of these effects is present in black holes.

\section{QPO correlations and frequency scalings}

We digress to note that the mechanism of QPO formation has previously been suggested to be common to sources as diverse as black holes and white dwarfs based on a linear relationship 
between two frequencies in LMXBs (Wijnands \& van der Klis 1999; Psaltis et al. 1999; Belloni et al. 2002), later extended to cataclysmic variables (Mauche 2002; Warner \& Woudt 2002). Because orbital motion around white dwarfs is accurately described by Newtonian gravity this seemed to rule out the few models, such as the so called relativistic precession model, in which all QPO frequencies can be related to general relativistic frequencies (Mauche 2002; Warner \& Woudt 2002). However, HF QPOs in black holes do not appear together with a lowfrequency QPO that would allow them to be placed on the correlation (Belloni 2005, private communication). In another view, the general scaling of frequencies with radius and mass, apparent in QPO sources, and anticipated in Kluźniak et al. (1990), suggests that QPOs and DNOs are an accretion disc phenomenon (Kluźniak et al. 2004a).

\section{A disc oscillation model for white dwarf DNOs}

It has been suggested that the twin HF QPOs in black holes correspond to two different modes of disc motion (e.g., radial oscillations and essentially vertical oscillations of the disc) which are in a 2:3 frequency ratio because they are excited by an internal resonance in the accretion disc (Kluźniak \& Abramowicz 2003). The first mode can modulate the emissivity of the disc, but the second mode is less likely to do so. However, in a black hole, axisymmetric vertical motion of the disc can modulate the $\mathrm{X}$-ray luminosity through gravitational lensing at the source, because the light trajectories are bent by differing amounts for different positions of the disc (Bursa et al. 2004). One of the two modes of oscillation occurs at the radial epicyclic frequency - or rather, its value at a certain position close to that of the pressure maximum of the accretion disc: Zanotti et al. (2003); Rubio-Herrera \& Lee (2005) - and the other at the vertical epicyclic frequency, in the same sense (Lee et al. 2004). We will call the epicyclic frequencies at this position in the disk "central". The two mode frequencies are different in strongfield Einstein's gravity, but they are equal in Newtonian $1 / r$ potential. The presence of two frequencies in white dwarfs cannot be explained by excitation of two distinct disc oscillation modes that are degenerate in frequency - harmonic overtones are a more likely cause in these dwarf novae ${ }^{1}$.

An analysis of the physical and statistical properties of the twin HF QPOs in neutron star systems has led to the suggestion that identical modes are resonantly excited in the neutron star and black hole systems (Kluźniak \& Abramowicz 2000, 2001; Abramowicz et al. 2003). It has also been pointed out that in neutron stars an additional source of disc excitation is present at its center, a non-axisymmetric rotating magnetosphere (Kluźniak et al. 2004a; Lee et al. 2004; Kato 2005), and that direct evidence of a resonance excited in this way is present in the transient accreting X-ray pulsar SAX J1808.43658 (Wijnands et al. 2003; Kluźniak et al. 2004b). Here, we note that the same source of excitation should be present in white dwarf systems, if a magnetospheric structure is present. Indeed, the observed period-luminosity relationship for DNOs

1 The radial overtones are harmonic as in a flute mode (Rezzolla et al. 2003).

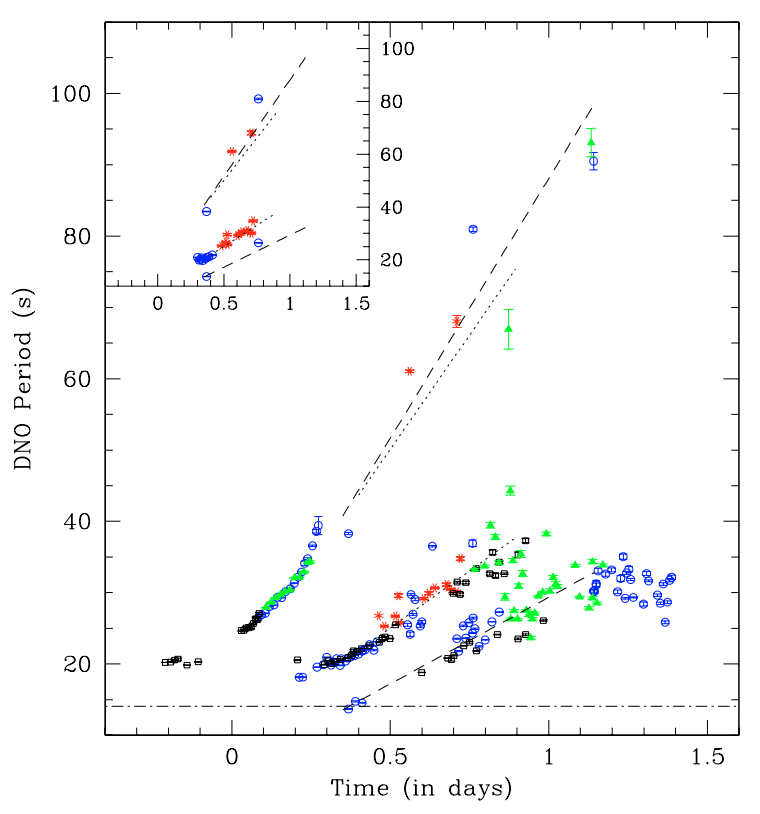

Fig. 1. The evolution of DNO periods at the end of normal and super outbursts in the dwarf nova VW Hyi. The different symbols indicate the various different kind of outbursts (short: asterisk, normal: open circles, long: open squares, and super outbursts: filled triangles). The dotted and dashed lines show the result of a least-squares fit to the first and second harmonic, respectively, and after a rescaling in period by a factor of two or three, respectively, they are replotted in order better to show the evolution of the DNO period. The zero of outburst phase is defined in Woudt \& Warner (2002). The inset highlights two observing runs in which the fundamental, first and second harmonic of the DNO period were present simultaneously. The horizontal dotted-dashed line illustrates the minimum DNO period (14.1 s) observed at maximum brightness. (From Warner \& Woudt 2005a).

prompted Paczyński (1978, see also Warner 1995) to suggest that magnetically channelled accretion was responsible, whereas King (1985) attributed DNOs to the presence of transient magnetic fields generated by turbulent dynamo in the white dwarf's outer layers.

We suggest two alternative explanations of the frequency evolution in Dwarf Nova Oscillations, based on extensive postoutburst observations of VW Hyi (Warner \& Woudt 2005a), see Fig. 1. The primary of the cataclysmic variable VW Hyi is a white dwarf of mass between 0.6 and $0.8 M_{\odot}$, which would correspond to a radius between 8 and $6 \times 10^{3} \mathrm{~km}$ (Schoembs \& Vogt 1981; Sion et al. 1997). The rotation period is not known, but spectral fits in quiescence suggest two components to the observed surface velocity $(v \sin i)$, about $400 \mathrm{~km} \mathrm{~s}^{-1}$ and about $4000 \mathrm{~km} \mathrm{~s}^{-1}$ (Godon et al. 2004), this could correspond to a spin of $\sim 10^{2} \mathrm{~s}$ or rotational period of an equatorial accretion belt of $\sim 10 \mathrm{~s}$. At maximum of outbursts DNOs are rarely seen, but when they are they are at $14.1 \mathrm{~s}$ (Warner \& Woudt 2005a).

Warner \& Woudt (2005a) point out that the frequency of DNOs decreases with the mass accretion rate and interpret this as the pushing out of the inner edge of the accretion disc by a magnetic field structure whose pressure increasingly overcomes the ram pressure of the accretion flow. In another 
interpretation of the DNO fundamental frequency, Warner \& Woudt (2005b) suggest that the frequency, linearly decreasing in time, can be understood as the period of rotation of an equatorial accretion belt magnetically coupled to the accretion disc, and possessing a non-axisymmetric structure (for the model, originally proposed by Paczyński 1978, see Warner 1995). We suggest that the evolution of the DNO frequency (plotted in Fig. 1) can be understood as a resonant response of the accretion disk to periodic perturbations by a magnetic field structure. In the first interpretation the perturbation period is fixed, but the disk eigenfrequency decreases. In the second interpretation the perturbation period decreases in time, while the eigenfrequency of the disk does not change.

An inspection of the data of Warner and Woudt (see Fig. 1) reveals that the frequency doubling does not occur at an arbitrary moment, but at that specific instant when the DNO frequency is about one half of its initial frequency. This can be understood if the observed frequency is that of an oscillation excited in the accretion disc by a perturbation related to the spin of the white dwarf, or of its equatorial layers. During the decay from outburst maximum either a permanent dipole structure of the white dwarf (Paczyński 1978; Lasota 2004), or a transient structure generated in a mechanism suggested by King (1985), affects the inner disc. There is an increasing mismatch of the perturbing frequency and the eigenfrequency of the disk, eventually leading to period halving, as described below.

Under the first interpretation, as the mass accretion rate drops (with the time elapsed from outburst maximum), the magnetic pressure pushes out the inner radius of the accretion disc, as in Warner \& Woudt (2005a), and the position of the pressure maximum in the disc moves out accordingly. The frequency of the fundamental mode decreases with the decreasing Keplerian frequency in the relevant parts of the $\operatorname{disc}^{2}$, and so do the frequencies of the overtones. The crucial point, is that the ratio of the overtones to the fundamental remains fixed in the process.

All the while, the white dwarf disturbs the disc periodically at its (the white dwarf) spin frequency. The coupling is sufficiently weak that the direct forcing frequency is barely present in the disc, instead, the disc responds to the perturbation at its own eigen-frequency (Kluźniak et al. 2004b; Lee et al. 2004), e.g., the "central" epicyclic frequency, which constantly decreases during the decay of the dwarf-nova outburst. Eventually the eigenfrequency of the disc drops to about one half of the perturbing frequency, and the perturbation can now resonantly excite the first harmonic. Once excited, this harmonic is preferentially maintained by the same perturbations, even as the disc eigenfrequency decreases. In VW Hyi at late times in the outburst, three frequencies are seen, in a 1:2:3 ratio (Fig. 1) The highest of these could be the second overtone, or it could be the non-linear beating of the strongly excited first overtone and the weakly present fundamental, at $f_{3}=f_{2}+f_{1}$. The difficulty of this model lies in the period of the white dwarf suggested by observations mentioned above (Godon et al. 2004), nearly

\footnotetext{
2 Once again, recall that in a Newtonian $1 / r$ potential the epicyclic frequencies are equal to the orbital frequency.
}

an order of magnitude larger than the $\sim 20$ s period required for this model of period halving.

In the second interpretation, the perturbing structure rotates at a variable rate along the equator, at rotation periods possibly as short as $14 \mathrm{~s}$, in agreement with the observations of Godon et al. (2004). The decreasing frequency would then reflect the decreasing rate of angular momentum accretion onto the white dwarf, but the relevant properties of the disc (such as its inner radius) would not vary strongly in the relevant parts of the outburst (which is consistent with magnetic moment assumed in the VW Hyi outburst model of Schreiber et al. 2004). In this case, if one assumes strong coupling of the boundary layer to the disc, we would expect (and suggest) that the oscillatory motion of the disc is well approximated by that of a forced nonlinear oscillator, i.e., the prime response would be at the forcing frequency of the boundary layer rotation. As this forcing rotation decreases, eventually a resonant response at a harmonic of the forcing frequency occurs, when it becomes equal to about one-half (and later one-third, for the second harmonic) of the accretion disc eigenfrequency.

We suggest that the observed DNO corresponds to the disc response. In support of this interpretation, we recall that a nonlinear resonance occurs in a certain frequency range. In particular, as the driver frequency decreases, the resonance turns on suddenly, and at a frequency higher than the eigenfrequency of the oscillator. We note that in the data of Fig. 1, the harmonics first appear at shorter periods (larger frequencies) than the shortest period of the fundamental, which was observed earlier in the outburst.

A similar mechanism cannot be invoked for black hole discs, not only for lack of a suitable perturbing agent. If in black holes the two HF QPOs in the 2:3 frequency ratio were also overtones, they could be excited in a range of frequencies (just as the DNO overtones are present over a range of frequencies, cf., Fig. 1). Instead, the black hole QPOs appear at fixed frequencies. This is consistent with two oscillatory modes of an accretion disc whose frequency ratio varies with the properties of the disc, and only for a certain state of a disc has the value 2:3, which can be excited by an internal resonance. For instance, the ratio of the vertical to radial epicyclic frequencies varies smoothly between unity and infinity, as the circle of maximum pressure in the disc moves in from very large radii to the radius of the marginally stable orbit.

When a monotonic decay occurs as in the neutron-star X-ray transient system Aql X-1 the (single) kHz QPOs are observed only during the so-called transition state close to maximum and then only short-timescale frequency-flux correlations are recorded (Maitra \& Bailyn 2004; Zhang et al. 1998). In general, in neutron-star X-ray binaries no QPOs are observed at the maximum and at the end of the outburst (e.g., Cui 2000). One should keep in mind, however, that VW Hyi is unique among CVs in having QPOs that are most prominent towards the end of the outburst.

\section{Conclusions}

The frequency evolution of the DNO oscillations, and their harmonic structure can be understood as the response of the 
disc oscillator to an external perturbation applied at a constant frequency while the disc eigenfrequency decreases with time, as its properties change. Alternatively, the DNO could be the forced response to a perturbation of steadily increasing period of a disc with a fixed eigenfrequency. The frequency drift is reminiscent of the $\mathrm{kHz}$ QPOs in low-mass X-ray binary neutron stars, where the disc is also perturbed at the stellar spin frequency. But unlike in persistent LMXBs, the mass accretion rate drops monotonically in the dwarf novae outbursts, and so does the fundamental frequency of the oscillator, allowing an apparent frequency doubling as the higher harmonics become resonantly excited.

The second high-frequency QPO in neutron stars does not have a counterpart in accreting white dwarfs. The HF QPOs in black holes cannot be excited by the spin of the (non-existent) central star, their frequencies are not variable and are in a definite $2: 3$ ratio, in agreement with the relativistic model of internal accretion disc resonance.

Acknowledgements. We are grateful to Tomaso Belloni for information about black-hole HFQPOs. We thank the anonymous referee for his helpful remarks and criticism. JPL was supported in part by a grant from the CNES. Research supported in part by KBN grant 2P03D01424.

\section{References}

Abramowicz, M. A., Bulik, T., Bursa, M., \& Kluźniak, W. 2003, A\&A, 404, L21

Abramowicz, M. A., \& Kluźniak, W. 2001, A\&A, 374, L19

Abramowicz, M. A., \& Kluźniak, W. 2004, AIP Conf. Proc., 714, (New York: AIP), 21

Belloni, T., Psaltis, D., \& van der Klis, M. 2002, ApJ, 572, 392

Bursa, M., Abramowicz, M. A., Karas, V., \& Kluźniak, W., ApJ, 617, L45

Cui, W. 2000, ApJ, 534, L31

Godon, P., Sion, E. M., Cheng, F. H., et al. 2004, ApJ, 612

Hameury, J.-M., King, A. R., \& Lasota, J.-P. 1985, Nature, 317, 597

Kato, S. 2001, PASJ, 53, 1

Kato, S. 2005, PASJ, 57, 4

King, A. R. 1985, Nature, 313, 291

Kluźniak, W., \& Abramowicz, M. A. 2000, PRL, submitted [arXiv:astro-ph/0105057]
Kluźniak, W., \& Abramowicz, M. A. 2001, Acta Phys. Pol. B, 32, 3605

Kluźniak, W., \& Abramowicz, M. A. 2003, 12 JGRG, ed. M. Shibata et al. (Tokyo: Tokyo University Press), 69

Kluźniak, W., Abramowicz, M. A., \& Lee, W.H. 2004a, AIP Conf. Proc. 714, (New York: AIP), 379

Kluźniak, W., Abramowicz, M. A., Kato, S., Lee, W. H., \& Stergioulas, N. 2004b, ApJ, 603, L89

Kluźniak,W., Michelson, P., \& Wagoner, R.V. 1990, ApJ, 358, 538

Lasota, J.-P. 2004, RMxAC, 20, 124

Lee, W. H., Abramowicz, M. A., \& Kluźniak, W. 2004, ApJ, 603, L93

Maitra, D., \& Bailyn, C. D. 2004, ApJ, 608, 444

Mauche, C. W. 2002, ApJ, 580, 423

McClintock, J. E., Remillard, R. A. 2004, in Compact Stellar X-ray Sources, ed. W. H. G. Lewin, \& M. van der Klis (Cambridge, New York: CUP), in press [arXiv: astro-ph/0306213]

Paczyński, B. 1978, Nonstationary Evolution of Close Binaries, ed. A. Żytkow, PWN, Warsaw, 89

Psaltis, D., Belloni, T., \& van der Klis, M. 1999, ApJ, 520, 262

Remillard, R. A., Muno, M. P., McClintock, J. E., \& Orosz, J. A. 2002, ApJ, 580, 1030

Rezzolla, L., Yoshida, Sh., \& Zanotti, O. 2003, MNRAS, 344, 978

Rubio-Herrera, E., \& Lee, W. H. 2005, MNRAS, 357, L31

Schoembs, R., \& Vogt, N. 1981, A\&A, 97, 185

Schreiber, M. R., Hameury, J.-M., \& Lasota, J.-P. 2004, A\&A, 427, 621

Sion, E. M., Cheng, F. H., Sparks, W. M., et al. 1997, ApJ, 480, L17

Strohmayer, T. E. 2001, ApJ, 552, L49

van der Klis, M. 2000, ARA\&A, 38, 717

Wagoner, R. W. 1999, Phys. Rep., 311, 259

Warner, B. 1995, Cataclysmic variable stars, Cambridge Astrophysics Series (Cambridge, New York: CUP)

Warner, B., \& Woudt, P. A. 2002, ASP Conf. Ser. 261 (San Francisco: ASP), 406

Warner, B., \& Woudt, P. A. 2005a, ASP Conf. Ser. 330 (San Francisco: ASP), 227

Warner, B., \& Woudt, P. A. 2005b, in preparation

Wijnands, R., van der Klis, M., Homan, J., et al. 2003, Nature, 424, 44

Woudt, P. A., \& Warner, B. 2002, MNRAS, 333, 411

Wijnands, R., \& van der Klis, M. 1999a, ApJ, 514, 939

Zanotti O., Rezzolla L., \& Font J. 2003, MNRAS, 341, 832

Zhang, W., Jahoda, K., Kelley, R. L., et al. 1998, ApJ, 495, L9 